\title{
Perfil sintomático de los pacientes con Enfermedad Renal Crónica Estadio 4 y 5
}

\author{
Daniel Gutiérrez Sánchez ${ }^{1}$, Juan P. Leiva-Santos² ${ }^{2}$ María José Macías López ${ }^{3}$, Antonio I. Cuesta Vargas ${ }^{4 *}$ \\ ${ }^{1}$ Fundación Cudeca. ${ }^{2}$ Hospital de Manacor. ${ }^{3}$ Servicio de Nefrología. Hospital Regional Universitario de Málaga. \\ ${ }^{4}$ Profesor de la Universidad de Málaga. Departamento de Fisioterapia. Instituto de Investigación Biomédico de \\ Málaga (IBIMA). Universidad de Málaga. España
}

\section{Resumen}

Introducción: Los pacientes con Enfermedad Renal Crónica Avanzada (ERCA) experimentan una gran variedad de síntomas que afectan negativamente la calidad de vida del paciente.

Objetivos: Determinar la prevalencia e intensidad de síntomas y analizar su asociación con el filtrado glomerular (FG) y la comorbilidad.

Material y Método: Estudio transversal en el que se describe la sintomatología del paciente con ERCA en diálisis y prediálisis y se realiza un análisis de la asociación de los síntomas con el FG y la comorbilidad. Para la evaluación de síntomas se utilizó la versión española modificada de la Palliative care Outcome Scale-Symptoms Renal (POS-S Renal), un cuestionario validado para evaluar la sintomatología en esta población. La comorbilidad fue evaluada con el índice de comorbilidad de Charlson modificado (ICCm).

Resultados: 180 pacientes con ERCA fueron incluidos en este estudio. Más del $44 \%$ de pacientes describieron debilidad, dolor, depresión y dificultad para dormir. Se encontraron diferencias significativas en ambos grupos para síntomas como la debilidad $(p=0.027)$, poca movilidad ( $p=0.018)$, somnolencia $(p=0.03)$, estreñimiento $(p=0.015)$ y piernas inquietas $(p<0.01)$. El declive de la función renal se asoció con la sintomatología $(p=0,04)$. No encontramos asociación entre la comorbilidad y la sintomatología $(p=0,15)$.

Correspondencia:
Antonio I. Cuesta Vargas
Instituto de Investigación Biomédico de Málaga (IBIMA)
Universidad de Málaga. Departamento de Fisioterapia
C./ Arquitecto Francisco Peñalosa
Ampliación Campus Teatinos. 29071 Málaga
E-mail: acuesta@uma.es

Conclusiones: Los pacientes con ERCA sufren una elevada carga de síntomas. La evaluación y monitorización sistemática de síntomas mediante herramientas como el POS-S Renal puede ser útil en el ámbito clínico y en investigación. La integración de los Cuidados Paliativos en los Servicios de Nefrología puede contribuir a la optimización del manejo sintomático en esta población.

PALABRAS CLAVE: enfermedad renal crónica avanzada; evaluación de síntomas; cuestionarios.

Symptomatic profile of patients with Chronic Kidney Disease Stage 4 and 5

\begin{abstract}
Introduction: Patients with Advanced Chronic Kidney Disease (ACKD) experience multiple symptoms that negatively affect the quality of life.
\end{abstract}

Aims: To determine the prevalence and severity of symptoms, and their association with the glomerular filtration rate (GFR) and comorbidities.

Material and method: A cross-sectional study to examine the prevalence of symptoms in patients with ACKD on dialysis and predialysis, and their association with GFR and comorbidities was carried out. Symptom data were collected using the Spanish modified version of Palliative care Outcome ScaleSymptoms Renal (POS-S Renal), a validated questionnaire to assess symptoms in this population. Comorbidity was collected and scored according to the modified Charlson Comorbidity Index (mCCI). 
Results: The study sample included 180 patients with ACKD. More than $44 \%$ of patients described weakness, pain, depression and difficulty sleeping. There was a significant difference in the prevalence of symptoms between groups for weakness $(p=0.027)$, poor mobility $(p=0.018)$, drowsiness $(p=0.03)$, constipation $(p=0.015)$ and restless legs $(p<0.01)$. There was no association between disease severity and symptoms $(p=0,04)$. There was no significant correlation between comorbidities and symptoms $(p=0,15)$.

Conclusions: Patients with ACKD experience a high level of symptom burden. Routine symptom assessment using tools such as the POS-S Renal can be useful in clinical and research settings. Integrating the principles of Palliative Care in Nephrology Services can be beneficial for optimizing the symptom management in this population.

KEYWORDS: advanced chronic kidney disease; symptoms assessment; questionnaires.

\section{Introducción}

La Enfermedad Renal Crónica (ERC) es un problema de salud pública con una elevada incidencia y prevalencia que además se espera que aumente en los próximos años ${ }^{1}$.En España la prevalencia de Enfermedad Renal Crónica Avanzada (ERCA), definida como ERC en estadio 4-5 con un filtrado glomerular (FG) $<30 \mathrm{ml} / \mathrm{min}$ durante al menos tres meses, supera los 1.000 pacientes por millón de habitantes ${ }^{1-3}$. El número de pacientes que reciben tratamiento renal sustitutivo (TRS) ha aumentado constantemente en los países desarrollados ${ }^{4}$.

Los pacientes con ERCA sufren una amplia variedad de síntomas físicos y psicológicos con un elevado coste en atención $n^{5}$. Desde los estadios iniciales de la ERCA, la sintomatología es variable y afecta negativamente a la calidad de vida ${ }^{6}$. Los síntomas más frecuentes que sufren estos pacientes son debilidad, prurito, estreñimiento, dolor, cambios en el patrón del sueño, ansiedad, disnea, náuseas, piernas inquietas y depresión $n^{7,8}$. Los trastornos emocionales como la ansiedad y la depresión están relacionados con niveles más altos de síntomas somáticos ${ }^{9}$. En este sentido, los profesionales sanitarios desempeñan un papel esencial en la evaluación y manejo de la sintomatología del paciente en ERCA ${ }^{10}$. Los síntomas no controlados en esta población contribuyen a un mayor sufrimiento y el manejo sintomático en las fases tempranas de la enfermedad es una prioridad ${ }^{10,11}$. La colaboración de los profesionales de las áreas de Nefrología y Cuidados Paliativos (CP) puede tener un efecto positivo en la calidad de vida de los pacientes y sus familias ${ }^{12}$. Por eso, la integración de los principios de los CP en las áreas de Nefrología puede ser beneficiosa para pacientes, cuidadores y profesionales ${ }^{12,13}$. En este sentido, el paciente con ERCA podría beneficiarse de los CP desde el diagnóstico de la enfermedad, durante las etapas de TRS, y especialmente en la retirada de diálisis, y en aquellos pacientes en los que se considere el manejo renal conservador $(M R C)^{14}$. En esta línea, se considera que el MRC es una opción razonable para aquellos pacientes con comorbilidad elevada, edad avanzada y que no desean iniciar la diálisis ${ }^{14}$.

La escasez de estudios realizados en España sobre prevalencia de síntomas en esta población impide comparar la sintomatología entre los diferentes grupos de tratamiento, por lo que este aspecto debe ser investigado en mayor profundidad ${ }^{15}$.

Los objetivos de este estudio fueron: (1) describir la prevalencia y la severidad de los síntomas en pacientes con ERC estadio 4 y 5 en diálisis y prediálisis, y comparar la sintomatología en ambos grupos, 2) explorar la asociación entre el FG y la prevalencia y severidad de la sintomatología; y (3) explorar la asociación entre la comorbilidad y la prevalencia y severidad de la sintomatología.

\section{Material y Método}

\section{Diseño del estudio}

Estudio de observación transversal. Todos los datos utilizados en este estudio se obtuvieron a partir de una amplia muestra de pacientes con ERC estadio 4 y 5. En este estudio, se realizó un análisis descriptivo de la prevalencia e intensidad de síntomas, la comorbilidad y el FG de los pacientes con ERCA, tanto en diálisis como en prediálisis. Además, se exploró la asociación de la prevalencia y severidad de los síntomas con el FG y la comorbilidad.

Los pacientes incluidos procedían del Servicio de Nefrología del Hospital Regional Universitario Carlos Haya de Málaga. Los datos del estudio fueron recogidos por DGS y MJML entre los meses de abril y septiembre de 2015. 
Los criterios de inclusión fueron: (1) pacientes adultos con ERC estadio 4-5 en prediálisis o diálisis desde hace más de tres meses, ya que se considera como tiempo de adaptación al tratamiento; y (2) pacientes hispanoparlantes. Se excluyeron los pacientes con deterioro cognitivo y aquellos menores de 18 años.

Los pacientes fueron informados sobre el estudio y posteriormente se obtuvo un consentimiento informado por escrito. Se recogieron datos sociodemográficos y clínicos durante las entrevistas que tuvieron lugar en la consulta ERCA y en la sala de diálisis.

\section{Variables estudiadas}

Sintomatología. Para la evaluación de la sintomatología se utilizó la versión española modificada de la Palliative care Outcome Scale-Symptoms Renal (POS-S Renal). La versión original de este instrumento fue diseñada para medir la sintomatología en pacientes con ERCA, y evalúa 17 síntomas $^{16}$. Esta versión original ha sido traducida y adaptada al español y ha demostrado ser un instrumento válido y fiable en la evaluación de la sintomatología en ERCA (diálisis y prediálisis) ${ }^{17}$. En este sentido, el cuestionario mostró unas propiedades psicométricas adecuadas en cuanto a validez estructural, fiabilidad test-retest y validez de criterio ${ }^{17}$. El análisis factorial exploratorio indicó una estructura de dos factores y un excelente ajuste para el modelo bifactorial (índice de ajuste comparado $=0,98$, error de aproximación=0,068) ${ }^{17}$. El análisis psicométrico mostró valores adecuados en términos de fiabilidad $(r=0,909$ para el factor $1, r=0,695$ para el factor 2 y $r=0,887$ para la puntuación total del cuestionario), consistencia interna ( $\alpha=0,78$ para el factor 1 y $\alpha=0,56$ para el factor 2), y validez de criterio con un instrumento validado que ha sido utilizado en esta población (MSAS-SF) $(r=0,860)^{17}$. Un nuevo ítem fue añadido a la versión española modificada del POS-S Renal (calambres) ${ }^{17}$. Como resultado, la versión española modificada de este cuestionario contiene 18 síntomas ${ }^{17}$.

FG. Se calculó utilizando la ecuación de la Chronic Kidney Disease Epidemiology Collaboration (CKDEPI $)^{18}$. Esta ecuación incluye variables como la creatinina sérica, la edad, el sexo y la raza ${ }^{18}$.

Comorbilidad. Para evaluar la comorbilidad se utilizó el índice de comorbilidad de Charlson modificado (ICCm), siendo éste un índice válido y fiable que ha sido ampliamente utilizado en población renal ${ }^{19}$. El ICC contiene 19 condiciones comórbidas, que se puntúan de 1 a 6, añadiéndose una puntuación de 1 por cada década a partir de los 40 años ${ }^{19}$. En esta versión modificada, el ítem "infarto agudo de miocardio" se ha sustituido por "enfermedad cardiovascular"19. Se considera que una puntuación en el ICCm mayor o igual a 8 es altamente predictiva de mortalidad ${ }^{19}$.

\section{Análisis estadístico}

Se realizó un análisis descriptivo de las variables sociodemográficas y clínicas de la población. Se utilizaron estadísticos de tendencia central (media \pm desviación estándar) para las variables continuas y porcentajes para las variables categóricas.

Para describir la sintomatología se utilizaron porcentajes e intervalos de confianza (IC) al 95\%. Se determinó la distribución de la muestra mediante la prueba de Kolmogorov-Smirnov (KS). Para estimar la relación entre el FG y la comorbilidad con la sintomatología se utilizó el coeficiente de correlación de Pearson. Para la comparación de medias entre los diferentes grupos se utilizó la prueba ANOVA de un factor. Se tomó el valor de $p<0,05$ como estadísticamente significativo. Los análisis se realizaron utilizando el software de análisis estadístico SPSS versión 20 .

\section{Resultados}

\section{Características de la muestra}

180 pacientes con ERC estadio 4 y 5 fueron incluidos en este estudio, de los cuales 124 pacientes pertenecían al subgrupo prediálisis y 56 al subgrupo diálisis (44 hemodiálisis y 12 diálisis peritoneal). Las características sociodemográficas y clínicas se muestran en la tabla 1. Los valores perdidos fueron menores al $2 \%$. La media del ICCm fue $6,1 \pm 2,5$ para el total de la muestra. Las principales condiciones comórbidas fueron: diabetes, cardiopatía isquémica, enfermedad vascular periférica y neoplasias. Se encontraron diferencias significativas en la puntuación total del ICCm entre ambos grupos $(p=0,02)$ y una tendencia a la significación para la diabetes $(p=0,054)$ y la enfermedad ulcerosa $(p=0,056)$. Las puntuaciones del ICCm y principales condiciones comórbidas se muestran en la tabla 2.

\section{Prevalencia y severidad de los síntomas}

Los síntomas más frecuentes fueron: debilidad $-68,7 \%$ (IC: $22-76 \%$ ), dolor- 53,1\% (IC: $45-61 \%$ ), depresión-45\% (CI: 36-51\%), dificultad para dormir 
Tabla 1. Características sociodemográficas y clínicas del grupo total y de los subgrupos.

\begin{tabular}{|c|c|c|c|c|}
\hline Características & Total & Prediálisis & Diálisis & p \\
\hline Pacientes, $n$ & 180 & 124 & 56 & \\
\hline Edad, años & $66,3(14,6)$ & $69,8(12,6)$ & $58,7(15,9)$ & $<0,001$ \\
\hline Género & & 0.031 & & \\
\hline Hombres \% & 65 & 70,2 & 53,6 & 0,031 \\
\hline Mujeres \% & 35 & 29,8 & 46,4 & 0,031 \\
\hline $\begin{array}{l}\text { FGe CKD-EPI } \\
\mathrm{ml} / \mathrm{min} / 1,73 \mathrm{~m}^{2}\end{array}$ & $13,7(16,9)$ & $17(5,7)$ & $6,6(2,6)$ & $<0,001$ \\
\hline $\begin{array}{l}\text { Tiempo en MRC o } \\
\text { diálisis, meses }\end{array}$ & $31,8(27,1)$ & $27,5(17,9)$ & $41,3(39,1)$ & 0,001 \\
\hline Causas de la ERC & & & & 0,240 \\
\hline Enfermedad vascular \% & 35 & 42,7 & 17,9 & 0,001 \\
\hline Nefropatía diabética \% & 13,9 & 14,5 & 12,5 & 0,719 \\
\hline Enfermedad glomerular \% & 8,9 & 6,5 & 14,3 & 0,088 \\
\hline Enfermedad poliquística \% & 6,7 & 5,6 & 8,9 & 0,416 \\
\hline Etiología desconocida \% & 11,7 & 12,1 & 10,7 & 0,791 \\
\hline Otras causas \% & 23,8 & 18,5 & 35,7 & 0,012 \\
\hline
\end{tabular}

- 44,4\% (IC: $37-53 \%$ ), problemas en la boca $-42,8 \%$ (IC: $35-50 \%$ ), cambios en la piel - 37,5\% (IC: $29-44 \%$ ), poca movilidad $-37,3 \%$ (IC: $29-44 \%$ ), poco apetito $-37,2 \%$ (IC: $28-43 \%$ ), estreñimiento $36,1 \%$ (IC: $29-44 \%$ ), ansiedad $-35,2 \%$ (IC: $27-41 \%$ ), prurito - 34,6\% (IC: $27-$ $42 \%$ ), disnea-33,5\% (IC: $26-41 \%$ ) y somnolencia - 31,3\% (IC: $23-37 \%$ ). La prevalencia de síntomas para el grupo total se muestra en la figura 1 .

Se encontraron diferencias significativas en la prevalencia de síntomas entre prediálisis y diálisis, para debilidad ( $p=0,027)$, poca movilidad $(p=0,018)$, somnolencia $(p=0,03)$, estreñimiento $(p=0,015)$ y piernas inquietas $(p<0,01)$. La comparación de la prevalencia de síntomas entre prediálisis y diálisis se muestra en la figura 2. El número medio de síntomas padecidos por paciente fue de $6 \pm 3,4$ de un máximo de 18 , con un rango de 0-16 síntomas por paciente.

Tabla 2. ICCm y prevalencia de condiciones comórbidas del grupo total y de los subgrupos.

\begin{tabular}{|c|c|c|c|c|}
\hline Características & Total & Prediálisis & Diálisis & $\mathbf{p}$ \\
\hline Pacientes, $\mathrm{n}$ & 180 & 124 & 56 & \\
\hline Cardiopatía isquémica \% & 22,8 & 25,8 & 16,1 & 0,151 \\
\hline Insuficiencia cardiac congestiva \% & 9,4 & 9,7 & 8,9 & 0,875 \\
\hline Enfermedad vascular periférica \% & 14,4 & 15,3 & 12,5 & 0,620 \\
\hline Enfermedad cerebrovascular \% & 8,3 & 9,7 & 5,4 & 0,334 \\
\hline Demencia \% & 0 & 0 & 0 & \\
\hline EPOC \% & 6,7 & 6,5 & 7,1 & 0,864 \\
\hline Enfermedad del tejido conectivo \% & 7, & 8,9 & 5,4 & 0,418 \\
\hline Enfermedad ulcerosa \% & 3,3 & 1,6 & 7,1 & 0,056 \\
\hline Enfermedad hepatica leve \% & 7,2 & 6,5 & 8,9 & 0,555 \\
\hline Diabetes mellitus \% & 10 & 12,9 & 3,6 & 0,054 \\
\hline Hemiplegia \% & 0 & 0 & 0 & \\
\hline Enfermedad renal moderada o severa \% & 100 & 100 & 100 & \\
\hline Diabetes con daño orgánico \% & 22.2 & 25 & 16,1 & 0,184 \\
\hline Tumor localizado, leucemia, linfoma o mieloma multiple \% & 21,1 & 22,6 & 17,9 & 0,475 \\
\hline Enfermedad hepática moderada o severa \% & 0,6 & 0,8 & 0 & 0,503 \\
\hline Tumor sólido secundario metástasico \% & 2,2 & 1,6 & 3,6 & 0,412 \\
\hline SIDA \% & 0,6 & 0,8 & 0 & 0,503 \\
\hline ICC & $6,1(2,5)$ & $5,3(2,2)$ & $5,8(2,7)$ & 0,02 \\
\hline
\end{tabular}




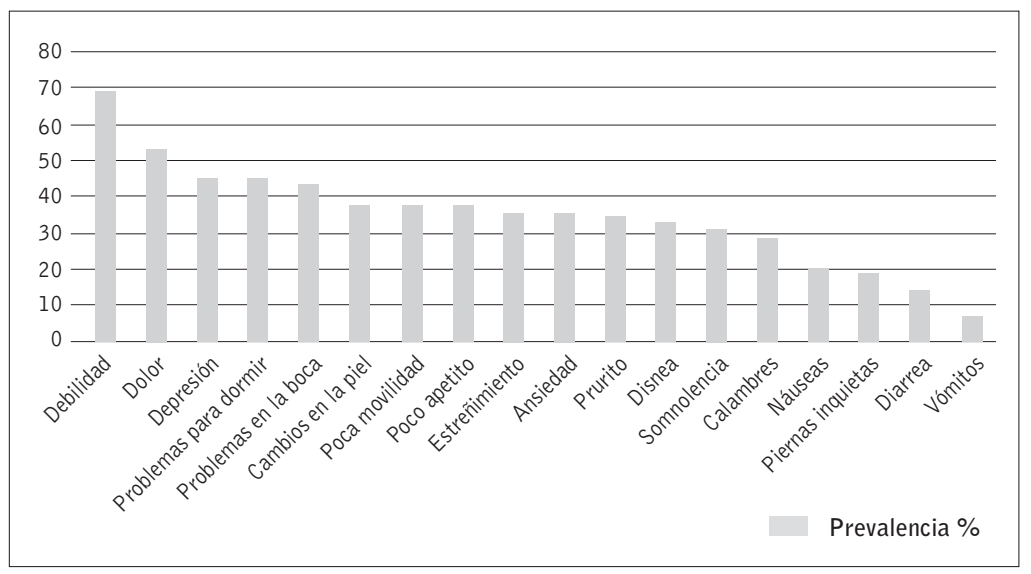

Figura 1. Prevalencia de síntomas de la muestra.

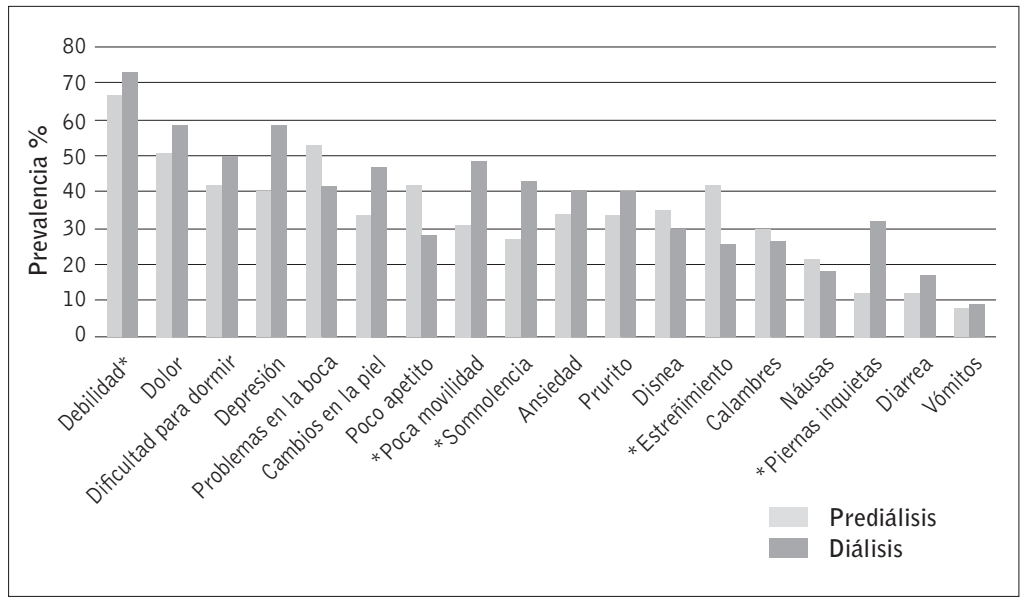

Figura 2. Prevalencia de síntomas de los subgrupos prediálisis y diálisis. Nota. Diferencia entre grupos: ${ }^{*} p<0,05$.

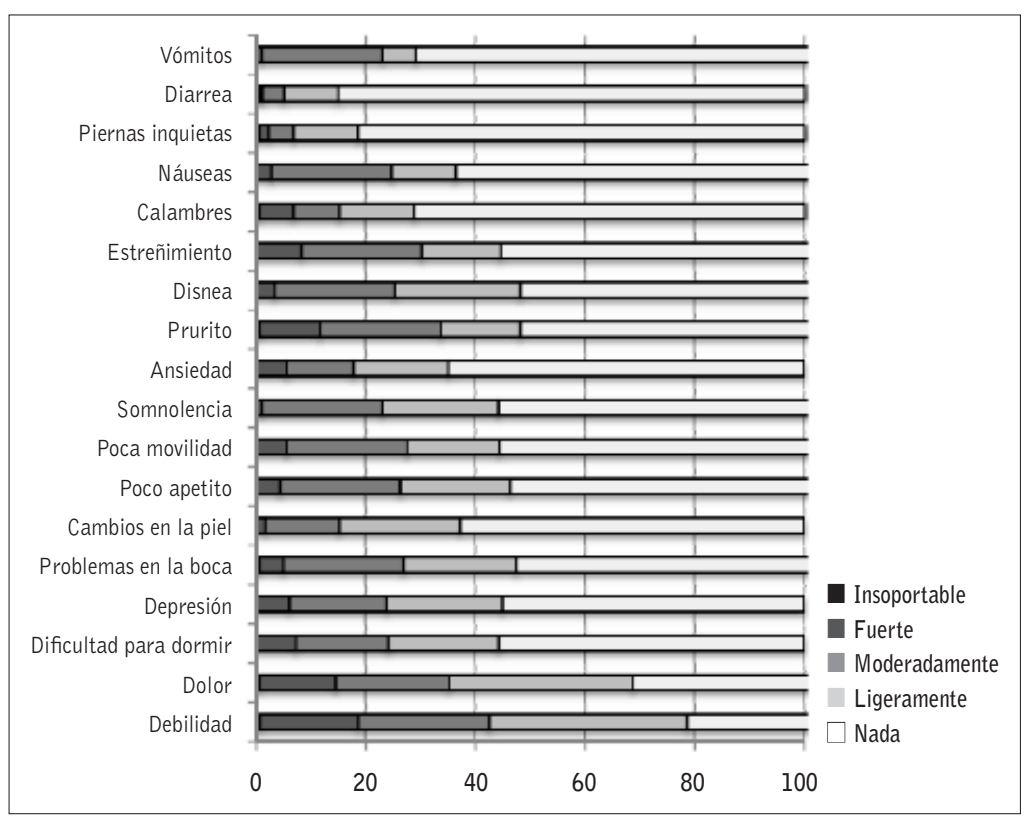

Figura 3. Severidad de los síntomas del grupo total.
Los síntomas más intensos (de severo a insoportable) fueron: debilidad (18,8\%), dolor $(14,6 \%)$ y prurito $(11,8 \%)$. La intensidad de los síntomas se muestra en la figura 3.

\section{Asociación entre la función renal y los síntomas}

Tras evaluar el efecto del declive de la función renal $(F G)$ en la carga y severidad de los síntomas, los resultados obtenidos indicaron que existía asociación entre el declive de la función renal y el número de síntomas $(r=-0,15, p=0,04)$, y por tanto el número de síntomas fue mayor en aqueIlos pacientes con un FG menor. Sin embargo, la función renal no se asoció con la severidad de la sintomatología $(r=-0,41$, $\mathrm{p}=0,58$ ).

\section{Asociación entre la comorbilidad y los síntomas}

También se evaluó el efecto de la comorbilidad sobre la carga y severidad de los síntomas. Los resultados indicaron que no hubo correlación significativa entre la comorbilidad y el número de síntomas padecidos por cada paciente $(r=0,11, p=0,15)$ y tampoco hubo correlación entre la comorbilidad y la severidad de los síntomas $(r=0,1, p=0,18)$.

\section{Discusión}

Este es el primer estudio que se realiza en España donde se analiza la prevalencia y severidad de síntomas en ERC estadio 4 y 5 y la correlación entre $F G$ y comorbilidad con la sintomatología en esta población, aunque la sintomatología de los pacientes con ERCA ha sido ampliamente estudiada en otras culturas ${ }^{7,8}$. Varios factores como la insuficiencia renal, la comorbilidad asociada o los factores asociados al TRS pueden influir en la en la sintomatología de estos pacientes ${ }^{14}$. Además, los síntomas en ERCA no se presentan de manera aislada, sino más bien en forma de grupos de síntomas ${ }^{20}$. 
Los resultados muestran que los pacientes con ERCA presentan una elevada prevalencia de síntomas y comorbilidad ${ }^{7,8}$. Síntomas como la debilidad $(p=0,02)$, poca movilidad $(p=0,01)$, somnolencia $(p=0,03)$, estreñimiento $(p=0,01)$ y piernas inquietas $(p=<0,01)$ presentaron una diferencia significativa en ambos grupos, siendo más frecuentes en pacientes con TRS (excepto el estreñimiento). Más del $44 \%$ de los pacientes presentaron debilidad, dolor, depresión y dificultad para dormir, encontrándose la debilidad y el dolor entre los síntomas más intensos. Estos síntomas son consistentes en los diferentes estudios y se asocian con una peor calidad de vida ${ }^{7,8}$.

La debilidad es muy frecuente en ERCA y se asocia con la carga global de síntomas, lo que sugiere que un manejo adecuado de la carga sintomática con un enfoque multidimensional podría disminuir la frecuencia e intensidad de este síntoma ${ }^{8,20}$. Varios factores, como los trastornos del sueño, la depresión, la anemia y el TRS pueden influir en la aparición de este sínto$\mathrm{ma}^{14,20}$. Los resultados indican que la debilidad fue el síntoma más prevalente y de mayor intensidad.

El dolor es un problema significativo en esta población ${ }^{21}$. Las causas del dolor en los pacientes con ERCA son diversas y están asociadas con la neuropatía periférica y los problemas vasculares-isquémicos, musculares y osteoarticulares, y además, la evidencia indica que este síntoma está relacionado con el insomnio y los síntomas depresivos ${ }^{21,22}$. En este estudio el dolor fue el segundo síntoma más prevalente e intenso.

En cuanto a los trastornos del sueño (apnea del sueño, insomnio, síndrome de piernas inquietas y somnolencia diurna), éstos son frecuentes en el paciente con ERCA y están interrelacionados con otros síntomas tales como la debilidad y la depresión ${ }^{23}$. Diversos factores como los trastornos psicológicos (depresión y ansiedad), el estilo de vida, el tratamiento y la comorbilidad, pueden influir en la aparición de estos trastornos $^{23}$. En este estudio la dificultad para dormir fue uno de los síntomas más intensos, y fue más común en pacientes en diálisis.

Respecto a los síntomas emocionales, los pacientes que reciben diálisis presentan una alta prevalencia de depresión que se asocia con una mala calidad de vida y otros síntomas físicos y emocionales ${ }^{6,24}$. EI TRS tiene un impacto en el bienestar físico y psicosocial y se traduce en un cambio significativo en el estilo de vida de muchos pacientes ${ }^{24}$. En este sentido, los resultados de este estudio muestran que la depresión fue más frecuente en el grupo diálisis, aunque no se encontró diferencia significativa entre los grupos de diálisis y prediálisis $(p=0,06)$. Esto sugiere la necesidad de una aproximación multidimensional con una intervención psicológica adecuada para esta población.

La sintomatología en ERCA está relacionada con el avance de la enfermedad renal ${ }^{14,25}$. En esta línea, los resultados de este estudio indican que los pacientes con ERCA en diálisis o prediálisis presentan una elevada carga de síntomas que está relacionada con el declive renal $(p=-0,04)$.

En cuanto a la asociación entre comorbilidad y el número y severidad de los síntomas, no se encontró correlación significativa. Estos hallazgos fueron comparables a los descritos por Brennan et $\mathrm{al}^{26}$.

Estudios previos indican que los síntomas experimentados en ERCA son similares a los encontrados en las etapas terminales de otras enfermedades, como el cáncer avanzado, la insuficiencia cardiaca congestiva, EPOC y SIDA, lo que indica que existe un patrón común de síntomas al final de la vida ${ }^{27,28}$. La atención paliativa en estas enfermedades puede ser relevante para mejorar la calidad de los cuidados, la calidad de vida y el manejo sintomático en estos pacientes ${ }^{29}$. Por lo tanto, los principios de los CP deberían integrarse en la atención de los pacientes con ERCA ${ }^{29}$. Esto es especialmente importante en España, donde la integración de los principios de los CP en los Servicios de Nefrología está en desarrollo.

Este estudio también muestra el valor de la versión española modificada del POS-S Renal como un instrumento que facilita al clínico una óptima evaluación de los síntomas en ERCA ${ }^{17}$. Este instrumento permite al clínico evaluar y monitorizar la amplia variedad de síntomas experimentados por estos pacientes ${ }^{17}$. Esto es especialmente importante en ERCA, donde instrumentos como el POS-S Renal pueden ser utilizados para evaluar y facilitar la introducción de intervenciones $^{30}$. Además, es útil disponer de instrumentos que puedan utilizarse en pacientes en diálisis y prediálisis en diferentes estadios de la ERC con el fin de controlar mejor los síntomas de los pacientes a medida que avanza la enfermedad ${ }^{17}$. Por lo tanto, la estandarización de herramientas de evaluación de síntomas como POS-S Renal puede ser útil tanto en clínica como en la investigación ${ }^{15-17}$.

Este estudio tiene varias limitaciones. Se trata de un estudio transversal que se ha realizado en un solo 
centro y por tanto los resultados pueden no ser generalizables. En este sentido, se necesitan estudios multicéntricos adicionales que usen una población más amplia. En este trabajo solo describimos la prevalencia e intensidad de la sintomatología, pero no realizamos una descripción detallada de cada síntoma. Además, los grupos prediálisis y diálisis no fueron homogéneos.

\section{Conclusiones}

Los pacientes con ERCA sufren una elevada carga de síntomas. En general, existe un perfil sintomático similar en prediálisis y diálisis. La debilidad es el síntoma más frecuente en ambos grupos. La integración de los CP en los Servicios de Nefrología puede contribuir a la optimización del cuidado y alivio del sufrimiento producido por los síntomas en esta población. En este estudio se pone de manifiesto la relevancia de la evaluación sistemática de los síntomas padecidos por el paciente con ERCA. Acorde a esto, clínicos e investigadores deberían utilizar herramientas válidas y fiables como el POS-S Renal para la evaluación de síntomas. Futuros estudios sobre el impacto de los síntomas en estos pacientes, así como las intervenciones necesarias para su óptimo manejo deberían ser considerados.

Recibido: 27 julio 2017

Revisado: 31 julio 2017

Modificado: 7 agosto 2017

Aceptado: 11 agosto 2017

\section{Bibliografía}

1. Otero A, de Francisco A, Gayoso P, Garcia F. Prevalence of chronic renal disease in Spain: results of the EPIRCE study. Nefrología 2010;30(1):7886.

2. K/DOQI Clinical practice guidelines for chronic kidney disease: evaluation, classification, and stratification. Am J KidneyDis. 2002;39(2 Suppl1):1-266.

3. KDIGO 2012 Clinical practice guideline for the evaluation and management of chronic kidney disease. KidneyInt (Suppl). 2013;3(1):1-308.

4. Guía para el tratamiento conservador en pacientes con Enfermedad Renal Crónica Avanzada, Govern de les Illes Balears, 2015.

5. Davison SN, Levin A, Moss AH, Jha V, Brown EA, Brennan $F$, et al. Executive summary of the KDIGO Controversies Conference on Supportive Care in Chronic Kidney Disease: developing a roadmap to improving quality care. Kidney international. 2015;88(3):447-59.1.

6. Abdel-Kader K, Unruh ML, Weisbord SD. Symptom burden, depression, and quality of life in chronic and end-stage kidney disease. Clinical journal of the American Society of Nephrology: CJASN. 2009;4(6):1057-64.

7. Murtagh FEM, Addington-Hall J, Higginson IJ. The prevalence of symptoms in end-stage renal disease: a systematic review. ACKD2007;14(1):82-99.

8. $O^{\prime}$ Connor NR, Kumar P. Conservative management of end-stage renal disease without dialysis: a systematic review. J Palliat Med. 2012 Feb;15(2):22835.

9. Perales-Montilla CM, Duschek S, Reyes-Del Paso GA. The influence of emotional factors on the report of somatic symptoms in patients on chronic haemodialysis: the importance of anxiety. Nefrologia. 2013 Nov 13;33(6):816-25.

10. Steinhauser KE, Christakis NA, Clipp EC, McNeilly M, McIntyre L, Tulsky JA. Factors considered important at the end of life by patients, family, physicians, and other care providers. JAMA. 2000 Nov 15;284(19):2476-82.

11. Manns B, Hemmelgarn B, Lillie E et al. Setting research priorities for patients on or nearing dialysis. Clin J Am SocNephrol. 2014; 9: 18131821.

12. Noble H, Kelly D, Rawlings-Anderson K, Meyer J. A concept analysis of renal supportive care: the changing world of nephrology. J Adv Nurs. 2007 Sep;59(6):644-53.

13. Arnold RM, Liao S. Editorial: renal palliative care: supporting our colleagues, patients, and family. J Palliat Med. 2006 Aug;9(4):975-6. 
14. Brown MA, Crail SM, Masterson R, Foote $C$, Robins $J$, Katz I, et al. ANZSN renal supportive care 2013: opinion pieces [corrected]. Nephrology (Carlton). 2013 Jun;18(6):401-54.

15. Gutiérrez Sánchez D, Leiva-Santos JP, SánchezHernández R, Gómez García R. Prevalencia y evaluación de síntomas en enfermedad renal crónica avanzada. Enferm Nefrol. 2015 Sep;18(3):228-36.

16. Murphy EL, Murtagh FEM, Carey I, Sheerin NS. Understanding symptoms in patients with advanced chronic kidney disease managed without dialysis: use of a short patient-completed assessment tool. Nephron Clinical Practice. 2009;111(1):74-80.

17. Gutiérrez-Sánchez D, Leiva-Santos JP, SánchezHernández R, Hernández-Marrero D, CuestaVargas AI. Spanish modified version of the palliative care outcome scale-symptoms renal: cross-cultural adaptation and validation. BMC Nephrol. 2016 Nov 18;17(1):180.

18. Montañés Bermúdez $R$, Bover Sanjuán J, Oliver Samper A, Ballarín Castán JA, Gràcia García $\mathrm{S}$. Assessment of the new CKD-EPI equation to estimate the glomerular filtration rate. Nefrologia. 2010;30(2):185-94.

19. Beddhu S, Bruns FJ, Saul M, et al. A simple comorbidity scale predicts clinical outcomes and costs in dialysis patients. Am $\mathrm{J}$ Med. 2000;108(8):609-13.

20. Almutary H, Douglas $\mathrm{C}$, Bonner A. Multidimensional symptom clusters: an exploratory factor analysis in advanced chronic kidney disease. J Adv Nurs. 2016 0ct;72(10):2389-400.

21. Davison SN. Pain in hemodialysis patients: prevalence, cause, severity, and management. Am J Kidney Dis. 2003 Dec;42(6):1239-47.

22. Davison SN, Jhangri GS. Impact of pain and symptom burden on the health-related quality of life of hemodialysis patients. JPSM. 2010 Mar;39(3):477-85.
23. Maung SC, El Sara A, Chapman C, Cohen D, Cukor D. Sleep disorders and chronic kidney disease. World J Nephrol. 2016 May 6;5(3):224-32.

24. Son Y-J, Choi K-S, Park Y-R, Bae J-S, Lee J-B. Depression, symptoms and the quality of life in patients on hemodialysis for end-stage renal disease. Am J Nephrol. 2009;29(1):36-42.

25. Tsai Y-C, Chiu Y-W, Hung C-C, Hwang S-J, Tsai $J-C$, Wang S-L, et al. Association of symptoms of depression with progression of CKD. Am J Kidney Dis. 2012 Jul;60(1):54-61.

26. Brennan F, Collett $G$, Josland EA, Brown MA. The symptoms of patients with CKD stage 5 managed without dialysis. Prog Palliat Care. 2015;23:267273.

27. Solano JP, Gomes B, Higginson IJ. A comparison of symptom prevalence in far advanced cancer, AIDS, heart disease, chronic obstructive pulmonary disease and renal disease. JPSM. 2006;31(1):5869.

28. Janssen DJA, Spruit MA, Wouters EFM, Schols JMGA. Daily symptom burden in end-stage chro $\neg$ nic organ failure: a systematic review. Palliat Med. 2008;22(8):938-48.

29. Tamura MK, Meier DE. Five policies to promote palliative care for patients with ESRD. Clin J Am Soc Nephrol 2013; 8: 1783-1790.

30. Perrone RD, Coons SJ, Cavanaugh K, Finkelstein $F$, Meyer KB. Patient-reported outcomes in clinical trials of CKD-related therapies: report of a symposium sponsored by the National Kidney Foundation and the US Food and Drug Administration. Am J Kidney Dis. 2013 Dec;62(6):1046-57. doi: 10.1053/j. ajkd.2013.07.004. 